> La dernière révision des lois de bioéthique encadrant la prescription d'un examen des caractéristiques génétiques a modifié les pratiques. Au-delà des droits du patient à recevoir une information claire et loyale, le prescripteur doit également l'informer, avant même la prescription de cette analyse, de ses devoirs vis-àvis des membres de sa famille en cas d'identification d'une anomalie génétique. À l'heure des techniques de séquençage à haut-débit et de leur application aux différentes spécialités médicales, une collaboration entre spécialistes d’organes et service de génétique médicale est fortement recommandée. <

\section{Aspects \\ réglementaires \\ du diagnostic \\ génétique en \\ France}

\author{
Cécile Zordan¹, Virginie Dorian ${ }^{1}$, Laetitia Jameau ${ }^{1}$, \\ Cyril Goizet ${ }^{1,2}$
}

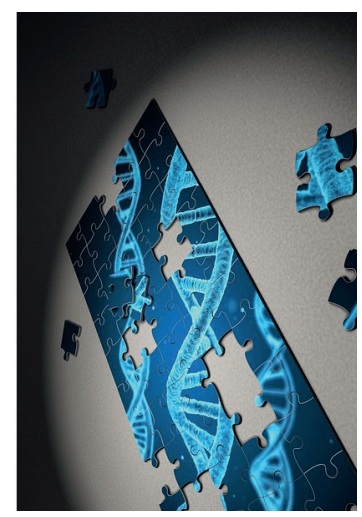

La France a été le premier pays au monde à se doter de lois de bioéthique dès 1994, le but étant d'encadrer les activités de génétique médicale clinique et biologique. Cette loi a été révisée plusieurs fois [1], la dernière datant de juillet 2011 [2], en s'appuyant largement sur les recommandations des comités d'éthique et des sociétés savantes. Ces révisions étaient devenues indispensables en raison des progrès continus de nos connaissances dans le domaine de la génétique, ayant amené à une évolution progressive de nos pratiques.

\section{Le conseil génétique}

Le conseil génétique est un acte médical destiné à un individu, malade ou non, à un couple ou à une famille. Une consultation de conseil génétique se déroule selon des modalités précises, à savoir :

- recueillir des informations médicales personnelles et familiales permettant de dessiner un arbre généalogique le plus précis possible, ce qui permet d'évoquer le mode de transmission de la maladie familiale;

- donner des explications quant aux caractéristiques cliniques de cette maladie, aux perspectives d'évolution des symptômes, des possibilités de traitements y compris symptomatiques, et des progrès de la recherche;
- expliquer le mode de transmission avec le pourcentage de risque de développer ou de transmettre la maladie ;

- informer sur les diverses possibilités de prévention faisant appel au diagnostic prénatal, au diagnostic préimplantatoire et au diagnostic présymptomatique.

Cette consultation de conseil génétique peut déboucher sur un prélèvement afin de réaliser une analyse génétique. Toutefois, la réalisation d'une analyse génétique n'est pas systématique à l'issue d'une telle consultation. Son indication doit répondre à l'existence d'une utilité clinique et au souhait de la personne chez qui elle va être réalisée [2, 3]. À l'opposé, un prélèvement dans le but d'une analyse génétique ne peut pas être réalisé sans une consultation de conseil génétique au préalable. En effet, toutes les informations et explications fournies lors de ce conseil génétique sont indispensables avant d'obtenir la signature d'un consentement éclairé.

Le conseil génétique n'est donc pas réservé à l'activité des médecins généticiens mais au contraire, tout médecin connaissant la situation clinique et ses conséquences personnelles et familiales, peut réaliser une consultation de conseil génétique et prescrire un examen à visée génétique. Le médecin prescripteur doit néanmoins être capable d'interpréter le résultat de l'analyse prescrite. II est fortement recommandé que les médecins spécialistes non généticiens travaillent en relation étroite avec une équipe de génétique clinique. Les conseillers en génétique, qui exercent une profession paramédicale apparue en France en 2005, sont également amenés à réaliser des actes de conseil génétique et à prescrire des analyses génétiques sous la responsabilité d'un médecin généticien [4]. 


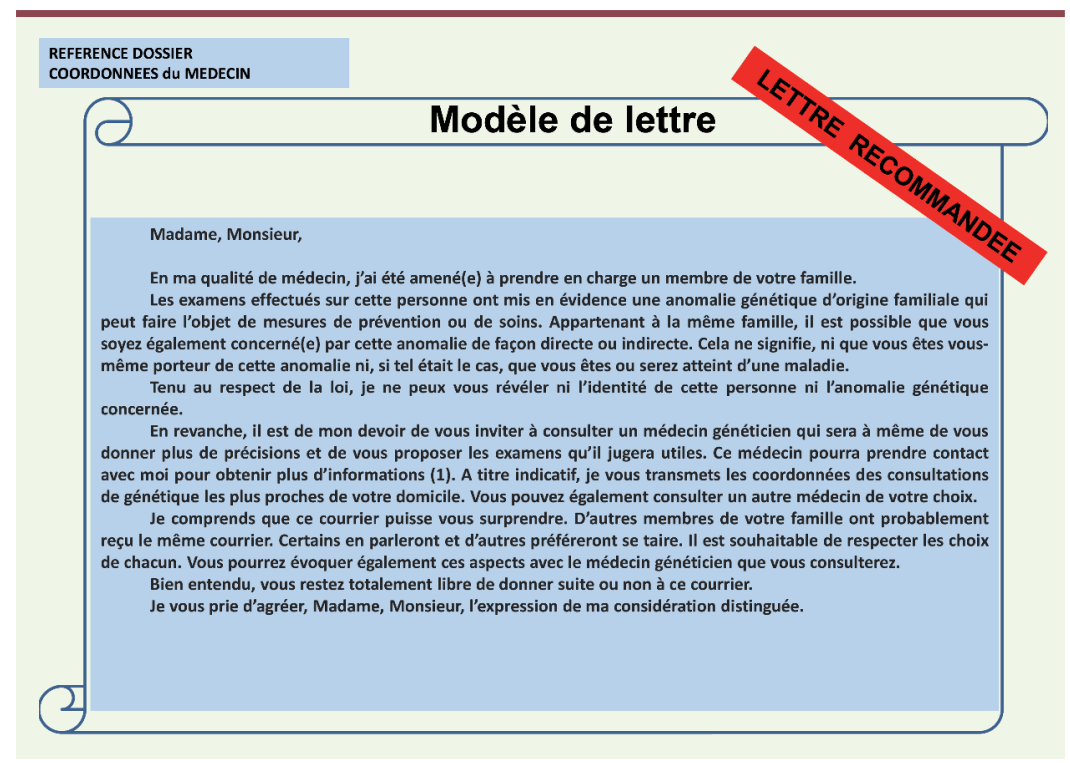

Figure 1. Modèle de lettre.

\section{Examens génétiques d'une personne à des fins médicales}

\section{Avant la prescription}

Il est indispensable de s'assurer de la bonne compréhension du patient à l'occasion de cette consultation qui reprendra tous les éléments du conseil génétique cités plus haut. II faudra aussi expliquer l'objectif de l'analyse génétique, les modalités de communication du résultat et les délais probables de leur obtention, les limites de l'analyse génétique prescrite, le risque éventuel de retrouver des données secondaires (en cas d'analyses génétiques à haut-débit), le droit au refus de l'examen ou à l'ignorance du résultat par l'individu, et enfin, les conséquences familiales engendrées par ce résultat dont l'obligation qui sera faite à ce moment-là d'informer ses apparentés. À l'issue de toutes ces explications, le consentement libre et éclairé peut alors être signé. Les formulaires de consentement ont d'ailleurs récemment évolué pour permettre de n'oublier aucun de ces points.

Le formulaire de consentement s'accompagne maintenant d'une attestation de consultation qui doit être signée par le prescripteur.

Une copie du consentement et une copie de cette attestation de consultation doivent être conservées par le prescripteur. Une copie doit être donnée au patient et une autre doit partir au laboratoire en charge de l'analyse génétique.

\section{La prescription}

Une prescription d'analyse génétique doit faire apparaître clairement l'analyse demandée, le motif de cette demande et surtout permettre l'identification du médecin prescripteur qui est celui qui sera amené à rendre en direct le résultat et qui donc, dans les hôpitaux, doit être un médecin senior, et non pas un interne ou un chef de clinique amené à changer régulièrement de lieu d'exercice.

Lorsqu'un test génétique est réalisé chez un sujet asymptomatique, dans le cadre d'un test présymptomatique, la prise en charge et le prélèvement à visée génétique doivent être exclusivement réalisés au sein d'une équipe pluridisciplinaire déclarée à l'Agence de Biomédecine pour cette activité.

\section{Rendu du résultat}

Le résultat d'un examen génétique doit être communiqué directement au patient par le prescripteur. Les modalités de communication de ce résultat ont été préalablement définies au cours de la consultation qui a donné lieu à la prescription. Ce rendu direct du résultat peut donc éventuellement se faire lors d'une consultation présentielle, éventuellement par contact téléphonique, mais pas par simple courrier.

Lors de ce contact direct, le résultat est communiqué par le prescripteur et doit être accompagné d'informations sur les conséquences pour l'individu lui-même ainsi que pour sa famille. Les modalités d'information à la parentèle doivent alors être recueillies, une copie du résultat de l'analyse doit être fournie au patient qui doit, dans le même temps, signer une attestation de rendu de résultat. Un document écrit résumant toutes ces informations doit être donné au patient, par exemple sous la forme du courrier médical qui lui est adressé en copie de celui envoyé au médecin traitant. L'existence et les coordonnées des associations de malades couvrant la maladie concernée doivent également être abordées.

\section{Information à la parentèle}

L'information à la parentèle correspond à la diffusion de l'information vis-à-vis d'une maladie génétique et d'un risque familial auprès des apparentés à risque [5]. Cette information à la parentèle est aujourd'hui obligatoire si le diagnostic d'une anomalie génétique grave [6] :

- peut faire l'objet de mesures de prévention ou de soins, permettant de diminuer un risque de décès prématuré ou de handicap. Ces actes de soins incluent par exemple des actes chirurgicaux, une prise en charge en kinésithérapie, une surveillance en imagerie pour prévenir l'apparition de tumeurs...;

- est utile au conseil génétique en vue d'un projet parental.

En fonction de l'anomalie génétique recherchée, le médecin détermine les apparentés qui seraient concernés par ce dispositif en fonction de l'arbre généalogique. L'information de la parentèle concerne le plus souvent les apparentés du premier degré (parents, 
enfants majeurs, frères et sœurs). En raison notamment de certains modes de transmission (lié à I'X, mitochondrial...) ou en cas d'impossibilité d'informer les apparentés du premier degré, il sera parfois nécessaire d'informer les apparentés du $2^{\mathrm{e}}$ degré voire de degrés supérieurs. D'autres paramètres peuvent être pris en compte (grossesse en cours, consanguinité...). Trois modalités d'information à la parentèle sont envisageables:

- Modalité $n^{\circ} 1$ : information directe de la famille. Si la personne exprime son souhait d'informer elle-même les intéressés ou certains d'entre eux, le médecin prescripteur le mentionne dans le dossier médical et précise l'identité des tiers qui seront informés directement par la personne. Pour l'aider à la diffusion de cette information, le médecin peut proposer des mesures d'accompagnement comme un document qu'elle pourra remettre à ses apparentés [5].

- Modalité $n^{\circ} 2$ : information via la procédure du décret $n^{\circ}$ 2013-527 [3]. Lorsque la personne ne souhaite pas informer elle-même les membres de sa famille, elle demande alors au médecin prescripteur de procéder à la transmission de cette information. Le prescripteur doit alors, par lettre recommandée, porter à la connaissance des membres de la famille potentiellement concernés l'existence d'une information médicale à caractère familial susceptible de les concerner, sans dévoiler le nom de la personne ayant fait l'objet de l'examen ni l'anomalie génétique ni les risques qui lui sont associés. Un modèle de lettre a été mis en disposition à l'occasion de la sortie du décret d'application (Figure 1) [7].

- Modalité $n^{\circ} 3$ : refus d'information par l'individu. Le médecin doit l'informer sur le risque de perte de chance, en termes de prise en charge médicale, que fait courir la personne qui refuse de transmettre l'information aux membres de sa famille concernés. II informe la personne que, selon la loi, elle est tenue d'informer sa parentèle des résultats des examens réalisés. En cas de non-respect de cette obligation, la responsabilité civile de la personne peut être engagée dans les conditions du droit commun. En cas de défaut d'information, l'individu pourrait être tenu responsable pour faute des éventuels dommages et pourrait être condamné par un juge à indemniser les préjudices (versement de dommages et intérêts) [5].

\section{Conclusion}

L'activité de génétique médicale occupe une place sans cesse grandissante dans les divers champs de la médecine. De nombreux spécialistes sont amenés de plus en plus régulièrement à pratiquer des consultations de conseil génétique avant de réaliser une analyse génétique chez leurs patients. Les lois de bioéthique imposent un cadre très rigoureux dans les étapes à accomplir avant la réalisation d'un test génétique, au moment du prélèvement et lors du rendu d'un résultat. Ces dispositions ont alourdi les conditions de réalisation de ces actes, nécessitant de prévoir un temps suffisant avant une prescription d'un acte génétique et au moment du rendu du résultat, ce qui est parfois incompatible avec l'organisation des différentes spécialités. La collaboration étroite des spécialistes d'organes avec un service de génétique clinique est très fortement recommandée en particulier pour aider à organiser au mieux l'information à la parentèle en s'appuyant sur les conseillers en génétique en exercice dans ces services. II faut en effet arriver au travers des diverses consultations à concilier divers enjeux comme le respect du secret médical et de la vie privée, la préservation du lien de confiance entre le médecin et l'individu qui vient le consulter, éviter de dissuader une personne malade de réaliser un examen à visée génétique tout en préservant à la fois son droit de ne pas savoir et son droit à ne pas lui-même diffuser l'information à sa parentèle. Il est indispensable de notifier par écrit dans le dossier médical les différentes étapes accomplies à l'occasion des consultations de conseil génétique précédant ou suivant un test génétique. Enfin, le développement des analyses du génome à haut débit invite à rester très humble par rapport au risque de découverte, par exemple, d'un variant de signification inconnue ne permettant pas une interprétation précise et claire des résultats, ou bien d'une donnée secondaire, c'est-à-dire d'un résultat qui n'était pas recherché mais qui pourrait avoir des conséquences importantes pour la santé future de l'individu. L'encadrement législatif de l'activité de génétique médicale sera ainsi très probablement amené à évoluer dans le futur au gré des progrès de nos connaissances. $\diamond$ Legal aspects of genetic testing for diagnostic purposes in France

\section{LIENS D'INTÉRÊT}

Les auteurs déclarent n'avoir aucun lien d'intérêt concernant les données publiées dans cet article.

\section{RÉFÉRENCES}

1. Loi du 6 août 2004 relative à la bioéthique (Art L.1131-1) : procédure de l'information médicale à caractère familial via l'Agence de la biomédecine.

2. Loi n 2011-814 du 7 juillet 2011 relative à la bioéthique modifiant le dispositif d'information de la parentèle dans le cadre d'un examen des caractéristiques génétiques (article L1131-1-2 du CSP).

3. Arrêté du 27 mai 2013 définissant les règles de bonnes pratiques applicables à l'examen des caractéristiques génétiques d'une personne à des fins médicales.

4. Loi de santé publique $n^{\circ} 2004-806$ du 6 août 2004 définissant la profession de conseiller en génétique (Art. L. 1132-1).

5. Décret $n^{\circ} 2013-527$ du 20 juin 2013 relatif aux conditions de mise en œuvre de l'information de la parentèle dans le cadre d'un examen des caractéristiques génétiques à finalité médicale.

6. Arrêté du 8 décembre 2014 définissant les règles de bonnes pratiques relatives à la mise en œuvre de l'information de la parentèle dans le cadre d'un examen des caractéristiques génétiques à finalité médicale.

7. Arrêté du 20 juin 2013 fixant le modèle de lettre adressée par le médecin aux membres de la famille potentiellement concernés en application de l'article R. 1131-20-2 du code de la santé publique. 